\title{
Stochastic Variability of Luminous Blue Variables
}

\author{
P. Abolmasov \\ Sternberg Astronomical Institute, Moscow State University, Moscow, Russia 119992; \\ Email: pavel.abolmasov@gmail.com
}

\begin{abstract}
Using the archives of the American Association of Variable Stars Observers and our own data, we analyse the long-term variability of several well-studied Luminous Blue Variables (LBVs) aiming on a general picture of stochastic variability of these objects. The power density spectra of all the selected objects may be generally described by a single power law contaminated by observational noise at higher frequencies. The slopes of the power-law component are close to $p=2$ (where $P D S \propto f^{-p}$, and $f$ is frequency) for strongly variable flaring objects like AG Car and significantly smaller $(p \sim 1.3)$ for $\mathrm{P}$ Cyg where brightness variation amplitude is $\lesssim 1^{\mathrm{m}}$ and dominated by slow low-amplitude variability. The slope holds for about two orders of magnitude in the frequency domain, though peaks and curvatures are present at $f \simeq 10^{-2} \div 10^{-3} \mathrm{~d}^{-1}$. We show that pseudo-photosphere approach to variability may explain the power-law shape of the variability spectrum at higher frequencies. However, the observed spectra are actually rather "red" than "brown": flux variations are correlated up to tens of years that is much longer than the characteristic refreshment time scales of the pseudo-photosphere. We propose that several stochastic noise components produce the power spectra of LBVs.
\end{abstract}

Keywords: stars: activity, stars: winds, outflows, stars: individual (P Cygni, eta Carinae, AG Carinae, Romano star, M33 V* V268 (=var C) )

\section{Introduction}

Flares from luminous blue variables (LBV) are studied for about four hundred years, since the 1600 flare of P Cyg (de Groot et al., 2001). However, these objects are rare and thus relatively unstudied. As variable objects, LBV are characterised by a complex hierarchy of variability timescales and 
amplitudes (Humphreys and Davidson, 1994). Usually, three empirical variability scales are distinguished:

1. microvariations, with amplitudes $\sim 0.1$ and relatively small variability timescales, from days to months

2. S Dor cycles, or eruptions (following Humphreys and Davidson (1994)) with amplitudes $\sim 1 \div 2^{\mathrm{m}}$, years to tens of years in length

3. giant eruptions with similar and larger (up to $\sim 100 y$ ) timescales and larger amplitudes $\left(\gtrsim 2^{\mathrm{m}}\right)$

Physically, giant eruptions may differ from the variations of the second type by significant changes in the bolometric luminosity (see discussion in section 5.2). Some authors like van Genderen et al. (1997a) propose division of the second type variability into normal and very long timescale $\mathrm{S}$ Dor cycles. Longer-timescale variations indeed seem to represent a separate mode of LBV variability, that is most evident in the XXth-century light curve of $\mathrm{P}$ Cyg (see next section) that is free from ordinary flares but dominated by microvariations superimposed over slow variations $\gtrsim 10 \mathrm{yr}$ in length.

Several well-studied LBVs exhibit periodic variability components. In particular, van Genderen et al. (1997b) mention a 1yr period for AG Car and $\mathrm{a} \sim 7 \mathrm{yr}$ period for $\mathrm{S}$ Dor. Probably, these periods do not correspond to any coherent process but rather reflect some characteristic timescales close to the duration timescale of a single flare.

$\eta$ Carinae is an exceptional case in this sense. The well-known 5-year period is stable on the timescales of tens of years that supports the idea that this object is indeed a (rather broad) binary (Damineli et al., 2008). Note however that the orbital separation of a massive binary with a 5-year period should be $a \simeq 2 \times 10^{14}\left(M / 100 \mathrm{M}_{\odot}\right)^{1 / 3}(\mathrm{~T} / 5 \mathrm{yr})^{2 / 3} \mathrm{~cm}$. Hydrostatic radii for LBV and related stars are $\sim 10^{12} \mathrm{~cm}$ that makes the expected effects of binary interaction on the central machine (-s) of $\eta$ Car very small. In this study, we find the broad-band PDS of $\eta$ Car quite similar to that of other flaring LBVs such as AG Car.

All the observed variability time scales of LBV stars lie between their dynamic and thermal times. Dynamic time scale is determined by the mean density of the stellar hydrostatic core. Outflowing matter can not take part in pulsations because the wind rapidly becomes supersonic. Straightforward estimate for the dynamic timescale as the free-fall time yields: 


$$
t_{d y n} \simeq 0.6\left(\frac{R_{\star}}{10^{12} \mathrm{~cm}}\right)^{3 / 2}\left(\frac{M_{\star}}{100 \mathrm{M}_{\odot}}\right)^{-1 / 2} \mathrm{~d}
$$

Due to period doubling (see for example Buchler (1993)) and instability of pulsational modes, lower-frequency modes are excited during pulsations. Probably, this effect is observed in "ordinary" hot supergiant stars, where microvariations occur at unexpectedly long time scales of several days (van Genderen, 1985). Flaring LBV variability is still much longer.

On the other hand, Kelvin-Helmholtz time scale for a typical hypergiant is:

$$
t_{K H} \sim \frac{G M}{R L} \simeq 2 \times 10^{4} \frac{M_{\star}}{100 \mathrm{M}_{\odot}}\left(\frac{R_{\star}}{10^{12} \mathrm{~cm}}\right)^{-1}\left(\frac{L_{\star}}{10^{6} \mathrm{~L}_{\odot}}\right)^{-1} \mathrm{yr}
$$

Unfortunately, these time scales are unreachable for modern observations, but may be, in principle, studied indirectly by observations of the ejecta, possibly light echoes from strong flares and using the statistical properties of LBV ensembles.

All the considered variability scales are somewhere in between and may correspond either to secular effects in dynamic-timescale evolution ("slow pulsations") or to effects in the expanding atmosphere that has, evidently, longer characteristic timescales than the hydrostatic core. Stellar wind, ascribed some velocity $v_{w}$, mass loss rate $\dot{M}$ and opacity $\kappa$, is characterised by the radius of the (pseudo-) photosphere $R_{p h} \simeq \kappa \dot{M} / 4 \pi v_{w}$ and corresponding time scale that has the physical meaning of the replenishment time for the matter in the wind:

$$
t_{r e f} \sim R_{p h} / v_{w} \simeq 2 \frac{\kappa}{\kappa_{T}} \frac{\dot{M}}{10^{-5} \mathrm{M}_{\odot} \mathrm{yr}^{-1}}\left(\frac{v_{w}}{100 \mathrm{~km} \mathrm{~s}^{-1}}\right)^{-2} \mathrm{~d}
$$

Here, $\kappa_{T} \simeq 0.4 \mathrm{~cm}^{2} \mathrm{~g}^{-1}$ is the Thomson cross-section for the scattering by free electrons in a hydrogen-rich gas. Real opacity is higher and contains some contribution of true absorption processes that may increase the characteristic time scales by about an order of magnitude (see, for example, Iglesias and Rogers (1996), bearing in mind that, for a typical $\operatorname{LBV}, \lg T \sim 4 \div 5$ and $\lg R \sim-5 \div-7$ for the inner wind). Despite the apparently small value of $t_{r e f}$, it is an important estimate for the characteristic variability time of an optically thick hot stellar wind. Longer time scales arise due to higher 
opacities Mass loss modulation during strong outbursts leads to even longer time scales, up to years.

Bolometric luminosity is not conserved during strong flares (see discussion in section 5.2). Besides this, modeling LBV photospheres provides evidence (de Koter et al., 1996) for more complicated nature of the variability of these objects, incorporating pulsations, mass loss variations and some additional mechanisms (such as unstable pulsational modes) leading to tremendous energy release during giant eruptions.

The overall power density spectrum (PDS) of LBV variability has not been considered as a whole. It is tempting to compare LBV stars to active galactic nuclei where different time scales of a single flicker noise were for a long time interpreted as physically distinct variability processes (see for example Terebizh et al. (1989)). Below we show that indeed for several LBV stars, broad-band power spectra have steep power-law shapes.

In this work we aim for the broad-band PDS of LBV variability. In the next section, we make a compilation of observational data on several reasonably well-studied LBV stars and present their PDS in the following section 3. A possible explanation for the observed broad-band PDS shapes is proposed in section 4. Results are discussed in section 5 .

\section{Observational Data}

We selected three well-studied and representative Galactic LBV stars. For these objects (see table 1), relatively long observational series exist, spanning periods of time in excess of 50 years. The data were taken from the archives of the American Association for Variable Star Observers. Earlier data are primarily visual magnitude estimates, therefore we use only visual magnitudes. We also checked that the data series do not have gaps longer than several months and binned the observational data points by five to diminish the effect of single erroneous estimates and the round-off effect connected to the low precision $(\sim 0 \mathrm{~m} 1)$ of visual magnitude measurements. Note that using a median filter would not diminish the round-off effect.

We also consider V-band light curves of comparable length obtained for two Hubble-Sandage variables in M33, Romano's star (the light curve itself is described in Sholukhova et al. (2002)) and Hubble-Sandage Variable C (Humphreys et al., 1988). 
Table 1: Objects selected for analysis. For Galactic objects, the numbers of data points are given after binning.

\begin{tabular}{lccc} 
Object ID & Variability Limits, mag & Time span & Number of Data Points \\
\hline \multicolumn{3}{c}{ Galactic objects } \\
P Cyg & $4.5 \div 5.6$ & $1917-08-13 . .2010-05-07$ & 3396 \\
$\eta$ Car & $4.5 \div 8.0$ & $1943-07-24 . .2010-07-19$ & 4637 \\
AG Car & $5.6 \div 8.9$ & $1939-12-05 . .2010-05-03$ & 2186 \\
& & \\
Romano's star & $16.1 \div 18.6$ & $1949-12-20 . .2009-11-08$ & 802 \\
Var C & $15.3 \div 17.9$ & $1961-09-13 . .2005-11-08$ & 635
\end{tabular}

\subsection{Notes on Individual Objects}

P Cyg

P Cyg is known for its outbursts in 1600 and later (de Groot et al. 2001). However, in the considered nearly 100-year time span it hardly shows any signatures of activity, except for rare short-lasting low-amplitude excursions (Markova et al., 2001). Variability amplitude is primarily contributed by low-frequency variability. Several periodicities, from 17 to 100 days, were reported (Kolka, 2001).

$\eta$ Car

During the considered time span, its behaviour is as well relatively quiet, especially if compared with its tremendous outbursts in the XIX century. In our sample, $\eta$ Car is the only proven binary. Its orbital period does not show up strongly in the optical variability, probably because of the large optical depth of the outflows in the optical. The variability pattern is dominated by the gradual increase of the optical luminosity.

$A G C a r$

Variability of this prototypical LBV star was considered in van Genderen et al. (1997c) together with that of $\eta$ Car. The object exhibits several strong flares. Periodicity of $\sim 370^{\mathrm{d}}$ (somewhat smaller than the characteristic flare duration time scale) is known to be present. 
Romano's Star, or V532 (M33)

One strong flare with an amplitude of about $2^{\mathrm{m}}$ and a couple of smaller maxima represent a typical picture of S Dor variability. The V-band data and data reduction process are described in Sholukhova et al. (2002). The object is hotter than all the other stars of the sample and reaches the spectral class of WN8 in its low/hot state (Polcaro et al., 2011; Maryeva and Abolmasov, 2010). Its spectrum in the quiet state $\left(\mathrm{BIa}^{+} \mathrm{e}\right)$ is similar to that of $\mathrm{P}$ Cyg.

$\operatorname{Var} C$ (M33)

Behaviour of this variable denoted as Variable C by Hubble and Sandage (1953) was studied by Humphreys et al. (1988), who conclude that the object behaves as a typical $\mathrm{S}$ Dor variable with a higher than average mass loss rate. The variable spends about a half of its time in a high and cool phase of the $\mathrm{S}$ Dor cycle. Amplitudes of the flares reach $2.5 \mathrm{~m}$ in the visual band. The data were provided by Alla Zharova (private communication).

\section{Analysis and Results}

All the time series are non-uniform but free from strong aliases, therefore we use extirpolation method (Press and Rybicki, 1989), where the initially non-uniform time series is interpolated upon a regular grid, to compute the Fourier spectra. Fourier transform for uniform series was performed using the fftw library (Frigo and Johnson, 2005). Software written in C and IDL was used for extirpolation and binning of the power density spectra (PDS). PDS were binned by 10 . We use relative normalization defined as follows:

$$
P_{j}=\frac{1}{\Delta T}\langle F\rangle^{-2}\left|\tilde{F}_{j}\right|^{2}
$$

where $\langle F\rangle$ is the mean flux, $\tilde{F}_{j}$ is the $j$-th component of digital Fourier transform of the flux $F$ (already interpolated over a regular grid), $\Delta T=N \Delta t$ is the total effective time span, $N$ is the number of data points, $j=0 . . N-1$, $\Delta t$ is the spacing of the regular grid. This normalisation has an evident physical meaning of the relative variability power in a unit frequency range. Its expectation does not depend on the time span considered and on data binning. Variability amplitude may be estimated by integrating the PDS over frequency domain and taking a square root. 
Table 2: PDS Fitting Results.

Galactic

M33

$\begin{array}{llll}\text { P Cyg } & \eta \text { Car } & \text { AG Car } & \text { V532 }\end{array}$

Power Law:

$\begin{array}{lccccc}N, 10^{-4} \mathrm{~d}^{-1} & 1.9 \pm 0.2 & 5.3 \pm 0.4 & 1.6 \pm 0.3 & 0.59 \pm 0.17 & 0.42 \pm 0.09 \\ \alpha & 1.17 \pm 0.02 & 1.31 \pm 0.01 & 1.56 \pm 0.04 & 1.63 \pm 0.05 & 1.86 \pm 0.05 \\ \chi^{2} / \text { DOF } & 549 / 168 & 1393 / 230 & 545 / 107 & 166 / 38 & 213 / 30\end{array}$

Lorentzian:

$\begin{array}{lccccc}N, \mathrm{~d}^{-1} & 38 \pm 4 & 770 \pm 30 & 1900 \pm 1200 & 1.23 \pm 1.07 & 1.51 \pm 0.18 \\ \gamma, 10^{-4} \mathrm{~d}^{-1} & 2.16 \pm 0.07 & 1.69 \pm 0.03 & 1.4 \pm 1.3 & 1.23 \pm 1.07 & 1.51 \pm 0.18 \\ \chi^{2} / D O F & 1602 / 168 & 2574 / 230 & 2167 / 107 & 169 / 38 & 227 / 30\end{array}$

Power Law with White Noise Component:

$\begin{array}{lccccc}N, 10^{-4} \mathrm{~d}^{-1} & 0.47 \pm 0.07 & 0.67 \pm 0.06 & 0.090 \pm 0.007 & 0.008 \pm 0.004 & 0.08 \pm 0.03 \\ \alpha & 1.43 \pm 0.03 & 1.75 \pm 0.02 & 2.12 \pm 0.01 & 2.34 \pm 0.01 & 2.14 \pm 0.07 \\ N_{W}, 10^{-4} \mathrm{~d}^{-1} & 45.2 \pm 4.9 & 139 \pm 6 & 302 \pm 15 & 550 \pm 20 & 500 \pm 100 \\ \chi^{2} / D O F & 536 / 167 & 1344 / 229 & 455 / 106 & 123 / 37 & 199 / 29\end{array}$

All the obtained PDS (see figure 1) have approximately power-law shapes with putative flattenings at lower $\left(\lesssim 10^{-4} \mathrm{~d}^{-1}\right)$ and higher $\left(\gtrsim 0.01 \mathrm{~d}^{-1}\right)$ frequencies. The lower-frequency turnovers are smoothed out by the binning in the frequency domain, but are better seen at finer binning. The data were fitted with power-law $\left(P D S=N \cdot f^{-\alpha}\right)$, Lorentzian $\left(P D S=N /\left(1+(f / \gamma)^{2}\right)\right.$, with zero resonance frequency) and power-law + white noise $(P D S=N$. $f^{-\alpha}+N_{0}$ ) models. Our choice of the third model is justified by existence of the observational errors that create an uncorrelated (white) noise component. Lorentzian function is relevant because it is expected to represent the PDS of a Poissonian sequence of exponentially decaying flares (and, approximately, rapidly-decaying flares of other shapes; see section 4.4). It indeed proves to be a reasonable fit for the objects that demonstrate strong flaring activity. Fitting results are given is table 2 .

$\mathrm{P}$ Cyg here is unique in its spectral shape that has the hardest power- 

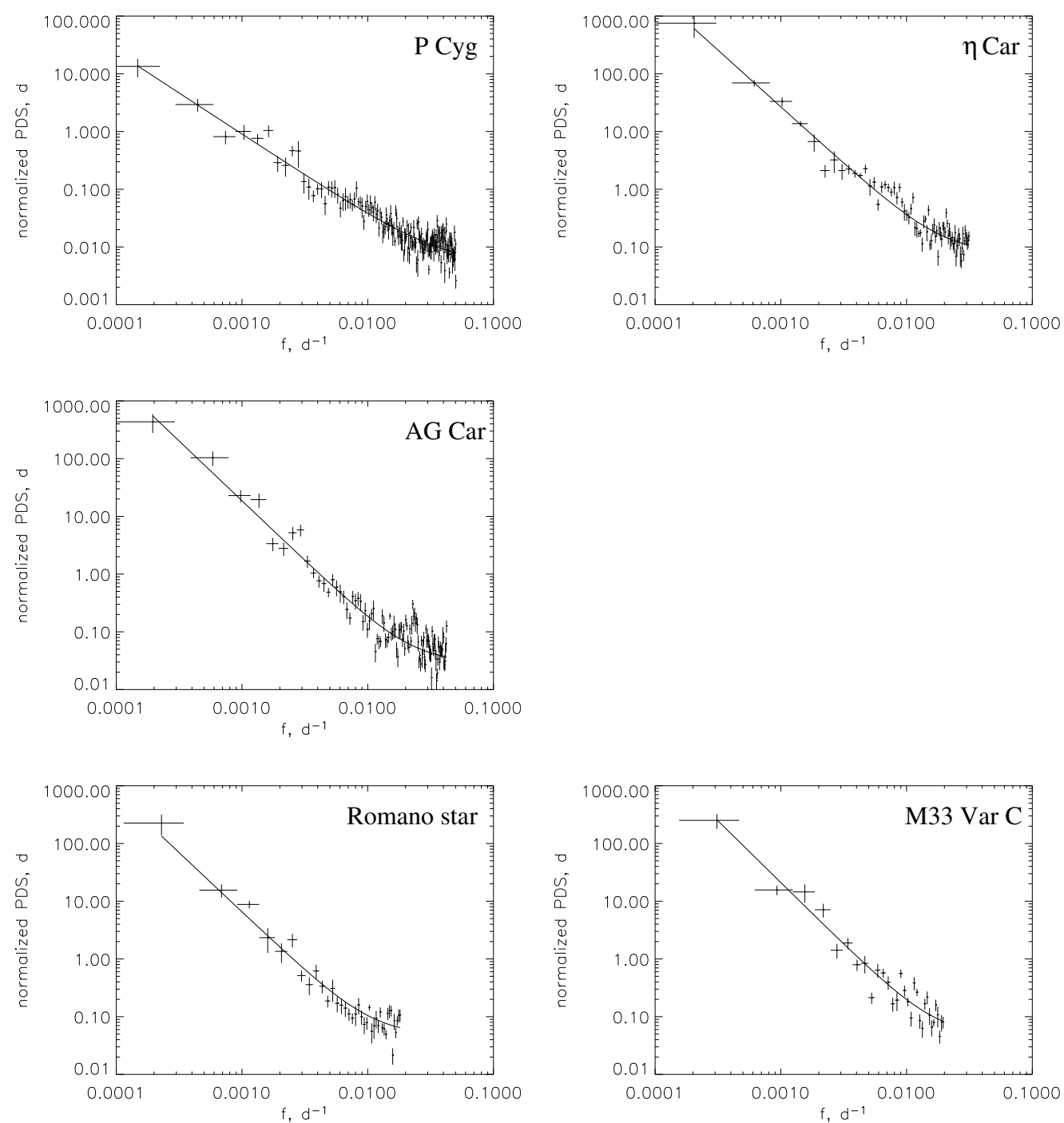

Figure 1: Power density spectra of the five variables fitted by the power-law + white noise model. 
law slope spanning a frequency range of two orders of magnitude. It is also unique in being the least active among all the objects. Other stars that exhibit S Dor cycles and outbursts have steeper spectral slopes close to 2 with possible indications for a low-frequency flattening. Average LBV power spectrum clearly has a power-law shape between tens of days to years or tens of years and possibly further toward hundreds of years. Gaining statistics and increasing the time spans to hundreds of years may allow to clearly resolve the turnover at about $f \sim 10^{-4} \mathrm{~d}^{-1}$ that definitely should take place because the light curve should remain reasonably uniform in time.

All the spectra exhibit curvatures and diffuse peaks that make all the fits rather poor $\left(\chi^{2} / D O F \sim 2 \div 5\right)$. The overall broad-band shape is, however, in good agreement with a single power law (with a high-frequency contribution from observational uncertainties) in all the cases.

\section{Variability Pattern of a Pseudo-Photosphere}

The idea of pseudo-photospheric nature of LBV cycles may be traced down to the note of Lamers (1987) who proposed that the primary source of the variability in these objects is the variability of the mass-loss rate. Below, we will assume that this variability is fast and uncorrelated (white noise in mass loss rate) and show that such variations indeed lead to a Brownianlike noise in the PDS, but with a turnover at a higher frequency. Lowerfrequency parts of LBV PDS require some additional mechanism of stochastic variability.

\subsection{Basic Assumptions}

Excluding brightest outbursts, variability of luminous blue variables is generally assumed to be purely spectral. According to Lamers (1987), the basic mechanism responsible for variable luminosities of LBV stars in the

optical is through variations in the mass loss rate resulting in variable pseudophotosphere radius and effective temperature.

In the relatively hot, relatively rarefied atmospheres of massive stars opacities are dominated by Thomson scattering, therefore the (radial) optical depth through the wind may be calculated as:

$$
\tau(R, t)=\frac{\kappa_{T}}{4 \pi} \int_{R}^{+\infty} \frac{\dot{M}(t-r / v(r)) d r}{r^{2} v(r)}
$$


Here, $v=v(r)$ is the velocity of the wind. Different true absorption processes also contribute to the opacity of the wind, but their effect depends on the temperature structure of the wind and on the effects of clumping. We neglect all the effects of true absorption though they are able to increase the opacity of the wind by a factor of several. This will mimic a larger mass accretion rate in the model. In its simplest form, the radius and effective temperature as a function of time may be estimated by equating the optical depth to some fixed value (below we use $\tau=2 / 3$ ). Equation (5) may be then solved for $r$.

\subsection{Numerical Simulations}

Six model light curves (see table 3) were calculated using a pseudophotosphere model with a blackbody photosphere defined by the condition of $\tau=2 / 3$. Bolometric luminosity was everywhere set to $L_{b o l}=3 \times 10^{39} \mathrm{erg} \mathrm{s}^{-1}$ that is close to its value for P Cyg as estimated by Pauldrach and Puls (1990). All the curves are 50 years in length and contain between 195 and 9774 data points. Model $\mathrm{F}$ was aimed on reproducing the basic variability properties of $\mathrm{P}$ Cyg. Others differ in the unperturbed mass loss rate $M_{0}$, mass-loss rate dispersion and wind velocity.

We use the semi-empirical $\beta$-law $v(r)=\left(v_{\infty}-v_{0}\right)(1-r / R)^{\beta}+v_{0}$ with $\beta=1$. Wind acceleration spatial scale $R$ is assumed identical to the hydrostatic inner radius $R$, and we set $v_{0}=2.5 \mathrm{~km} \mathrm{~s}^{-1}$ everywhere. Wind velocity at infinity was fixed, while the mass loss rate experiences log-normal variations that we assume uncorrelated on the time scales of interest. Of course, real stellar winds have variable velocities, but simultaneous variations of wind velocity and mass loss rate do not change the picture qualitatively. Variable wind velocity would however lead to formally divergent solutions for density distribution. In real winds, shock waves are expected to be formed. Hence the effects of variable wind velocity should be considered together with deviations from spherical symmetry and quasi-stationarity. Log-normality of the mass-loss rate distribution is a useful assumption because is allows to easily account for dramatic changes in the value of $\dot{M}$ without producing unphysical negative values. If the mass-loss rate varies on dynamical time scales, the wind will work as an integrator, making the observed power density spectrum softer than the spectrum of $\dot{M}$ variations. For most of the models, we assume the mass loss rate logarithm dispersion $\sigma(\ln \dot{m})=2$ that corresponds to about a factor of 7 change in the mass loss rate itself and may 
Table 3: Light Curve Simulations

\begin{tabular}{lcccccc} 
Model ID & A & B & C & D & E & F \\
& & & & & & \\
\hline$\dot{M}_{0}, \mathrm{M}_{\odot} \mathrm{yr}^{-1}$ & $10^{-4}$ & $10^{-4}$ & $10^{-5}$ & $10^{-4}$ & $10^{-4}$ & $10^{-5}$ \\
$\sigma(\ln \dot{m})$ & 2 & 2 & 2 & 3.5 & 1 & 1 \\
$v / 100 \mathrm{~km} \mathrm{~s}^{-1}$ & 1 & 0.5 & 1 & 1 & 2 & 2 \\
$R_{0}, 10^{12} \mathrm{~cm}$ & 28 & 14 & 3.7 & 28 & 55 & 3.7 \\
No of points & 3910 & 1955 & 9774 & 195 & 1955 & 5585 \\
$M_{V}$ range, mag & $-6.6 . .-10.0$ & $-6.7 . .-10.0$ & $-5.7 . .-8.2$ & $-6.1 . .-10.0$ & $-6.6 . .-10.0$ & $-5.7 . .-7.9$ \\
$\left\langle M_{V}\right\rangle$, mag & -8.2 & -9.0 & -6.0 & -9.0 & -9.0 & -6.0 \\
$\sigma\left(M_{V}\right)$, mag & 0.5 & 0.5 & 0.24 & 0.67 & 0.5 & 0.22 \\
$N\left(t_{\text {dyn }}<t_{\text {diff }}\right), \%$ & 0.2 & 0 & 0 & 4 & 0.2 & 0
\end{tabular}

account for the observed $\sim 1 \div 2^{\mathrm{m}}$ variations of $\mathrm{S}$ Dor cycles. Models $\mathrm{D}$ and E illustrate the effect of variable amplitude of mass loss variations.

Inner (hydrostatic) radius was everywhere set to $2 \times 10^{12} \mathrm{~cm}$, the scale height of the $\beta$ law for velocity is ascribed the same value.

In figure 2, we show the simulated light curves in the Johnson $\mathrm{V}$ band. Corresponding power density spectra are shown in figures 3 and 4 together with modified power law and Lorentzian approximations. Modified power law is defined as follows:

$$
F_{M P L}=N f^{p} \exp (-T f)+N_{0}
$$

This spectral law differs from the power law + white noise model used above by an exponential decay factor with characteristic time $T$, close to the wind replenishment time. If the mass loss is uncorrelated at higher frequencies, small fast variations should have a flat spectrum as well (see section 4.3.

Basic parameters of the light curves and fitting results are given in tables 3 and 4 , respectively. $N\left(t_{d y n}<t_{d i f}\right)$ in the table is the fraction of time when dynamical timescale defined as $t_{d y n}=R_{0} / v$ is smaller than the radial diffusion time scale defined as $t_{\text {diff }}=\tau R_{0} / c$, where $\tau$ is the total optical depth throughout the wind. If this inequation is satisfied, the assumption of instantaneous energy transfer vital for the pseudo-photosphere approximation is violated (see section 5.2). Power density spectra of the simulated light curves are shown in figures 3 and 4 

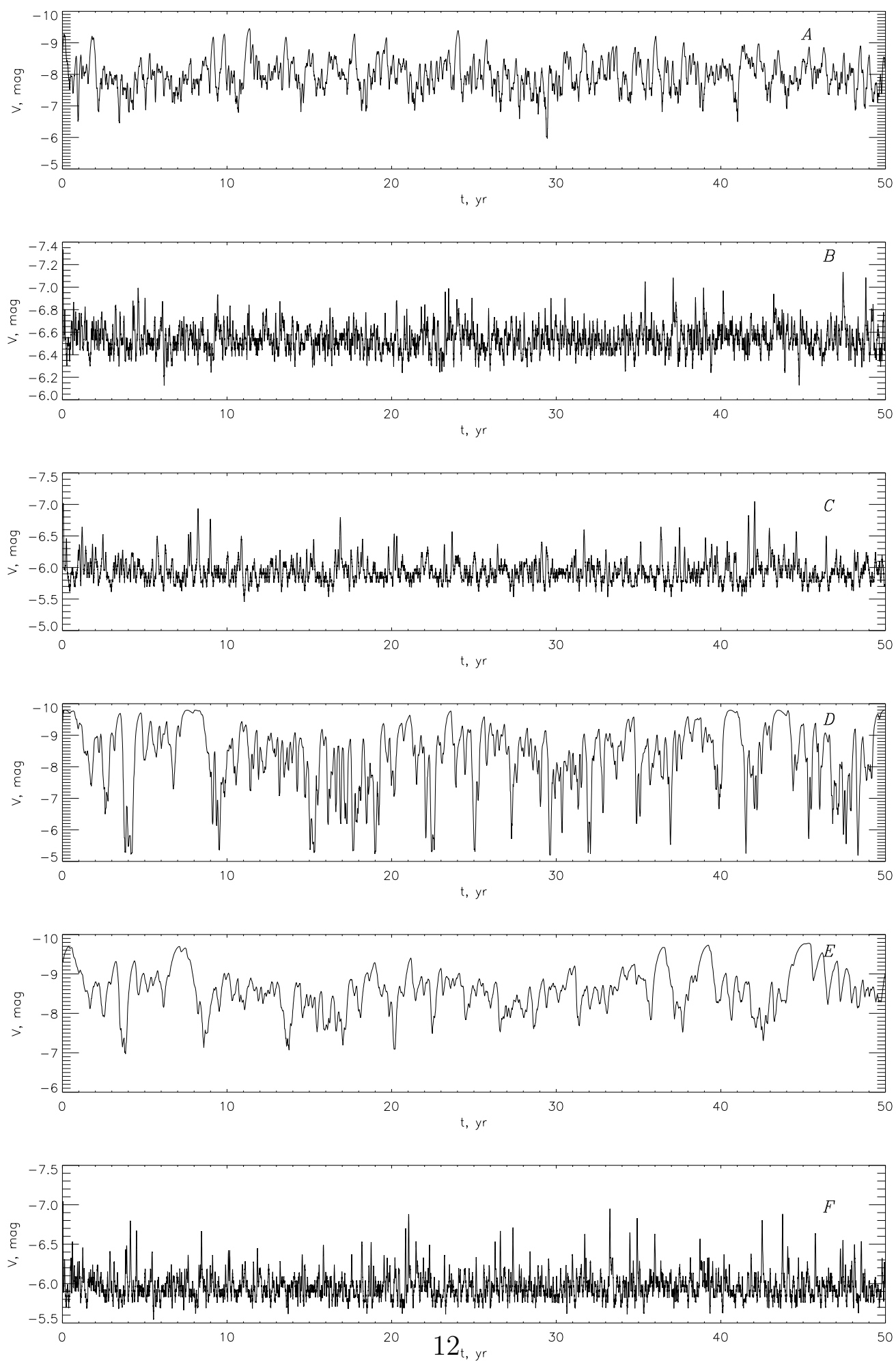

Figure 2: Simulated light curves. 
Table 4: PDS of the Simulated Light Curves

\begin{tabular}{lcccccc} 
Model ID & A & B & C & D & E & F \\
\hline \multicolumn{7}{c}{ Lorentzian: } \\
$N, \mathrm{~d}$ & $30 \pm 1$ & $0.468 \pm 0.007$ & $0.91 \pm 0.09$ & $120 \pm 10$ & $42 \pm 2$ & $1.2 \pm 0.1$ \\
$\gamma, 10^{-3} \mathrm{~d}^{-1}$ & $1.44 \pm 0.05$ & $3.92 \pm 0.19$ & $14 \pm 3$ & $1.2 \pm 0.3$ & $0.9 \pm 0.1$ & $6.48 \pm 0.05$ \\
$\chi^{2} / D O F$ & $2527 / 115$ & $1304 / 298$ & $1299 / 291$ & $55 / 24$ & $947 / 27$ & $1700 / 333$
\end{tabular}

Modified Power Law:

$\begin{array}{lcccccc}N, \mathrm{~d} & 550 \pm 40 & 2 \pm 4 & 40 \pm 40 & 284 \pm 3 & 104 \pm 1 & 12 \pm 7 \\ T, \mathrm{~d} & 361 \pm 8 & 100 \pm 28 & 160 \pm 20 & 614 \pm 17 & 8.55 \pm 5 & 103 \pm 11 \\ p & 0.41 \pm 0.03 & 0.3 \pm 0.2 & 0.46 \pm 0.16 & 0.00 \pm 0.01 & 0.00 \pm 0.01 & 0.41 \pm 0.10 \\ N_{0}, \mathrm{~d} & 17 \pm 9 & 0.631 \pm 2 & 1 \pm 3 & 0.6 \pm 0.2 & 110 \pm 10 & 1 \pm 2 \\ \chi^{2} / D O F & 1161 / 113 & 1128 / 296 & 1443 / 289 & 57 / 22 & 173 / 25 & 1362 / 331\end{array}$
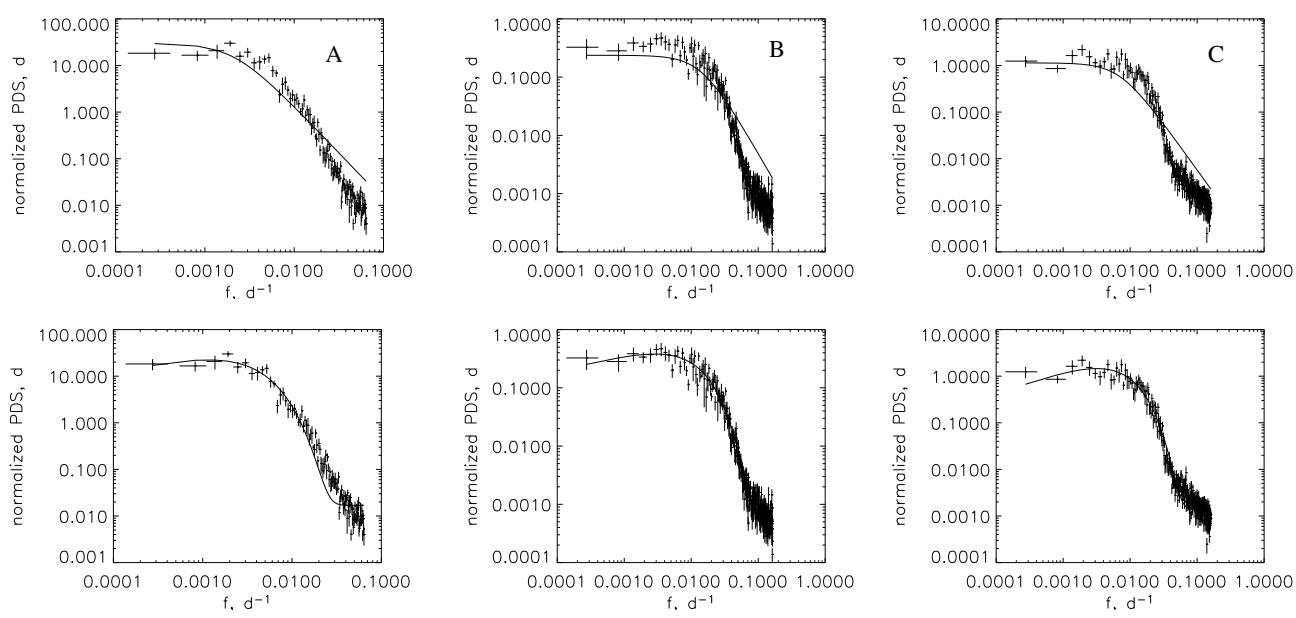

Figure 3: PDS fitting by Lorentzian (upper panels) and modified power law (lower panels) models for simulated light curves, models A-C. 

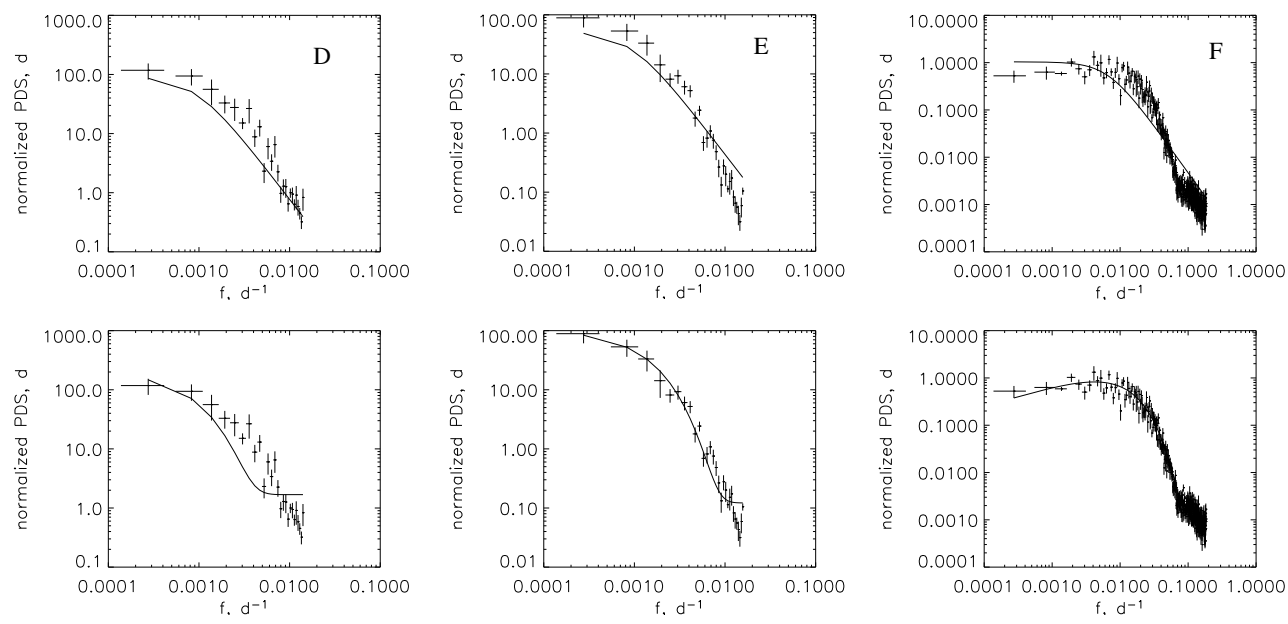

Figure 4: PDS fitting by Lorentzian (upper panels) and modified power-law (lower panels) models for simulated light curves, models D-F.

\subsection{Approximate Solution for Small Variations}

For the linear pseudo-photosphere described by the equation (5), it is easy to find the PDS for $\tau$ in the assumption $v=$ const. For small variations, $\tau$, $r(\tau)$ and the resulting luminosity will have identical spectral shapes.

Let $R_{0}$ be the radius corresponding to the photospheric radius calculated for $\dot{M}_{0}$ :

$$
R_{0}=\frac{3 \kappa_{T} \dot{M}_{0}}{8 \pi v}
$$

If $\dot{M}(t)$ is uncorrelated noise with the mean of $\dot{M}_{0}$ and dispersion $\dot{M}_{0} D \dot{m} \ll$ $\dot{M}_{0}^{2}$, the resulting PDS will be identical to the spectrum of the equivalent $\delta$ function response:

$$
\tau_{\delta}=\frac{2}{3} \sqrt{D \dot{m}} \frac{1}{t^{2}}
$$

Here, $t>R_{0} / v$, because only visible matter influences the opacity. Fourier image:

$$
\begin{aligned}
\tilde{\tau}(f) & =\frac{4 \pi}{3} \sqrt{D \dot{m}} f \int_{2 \pi f R_{0} / v} \frac{e^{-i \eta}}{\eta^{2}} d \eta= \\
& =\frac{4 \pi}{3} \sqrt{D \dot{m}} f\left(-\frac{\cos a}{a}-\operatorname{si}(a)+i\left(\frac{\sin a}{a}+\operatorname{ci}(a)\right)\right),
\end{aligned}
$$


where integral sine and cosine functions (si and ci) are defined as integrals from $a=2 \pi f R_{0} / v$ to positive infinity. Finally, power spectrum in the linear case behaves as:

$$
\begin{gathered}
P D S \propto D \dot{m} f^{2}\left(a^{-2}+2 a^{-1}(\cos a \operatorname{si} a+\sin a \operatorname{ci} a)+\mathrm{ci}^{2} a+\mathrm{si}^{2} a\right) \propto \\
\propto \begin{cases}f & f \ll v / 2 \pi R_{0} \\
1 & f \gg v / 2 \pi R_{0}\end{cases}
\end{gathered}
$$

The resulting PDS should be peaked, similar to the (6) law with $p=$ 1. The predicted power spectrum is vastly different from the quiet-state variability spectrum of LBVs.

\subsection{PDS in the Flaring Case}

The strongly-variable $\mathbf{D}$ model has a PDS qualitatively similar to those of strongly variable objects like AG Car. In fact, any source exhibiting smooth light variations during individual uncorrelated flare events is expected to have flat spectrum at the smallest frequencies and a Brownian-shaped $\propto f^{-2}$ noise in the opposite limit $(f \rightarrow \infty)$. If one considers a flare of duration $t_{f}$ (smooth in its maximum but with rapid rise and decay), its PDS shape in the high-frequency limit may be estimated as:

$$
|\tilde{F}(f)|^{2} \propto\left|\int_{-t_{f} / 2}^{t_{f} / 2} l(t) e^{-i 2 \pi f t} d t\right|^{2} \propto \frac{\sin ^{2}\left(\pi f t_{f}\right)}{f^{2}} \sim f^{-2}
$$

This approximation works for $f \gg 1 / t_{f}$. In the opposite frequency limit, variability is a Poissonian series of short events that implies white noise. The spectral slope changes somewhere around $f \sim 1 / t_{f}$, and the exact shape of the knee in the PDS depends on the shape of a single flare. The best known example is the Lorentzian spectrum for exponential flares.

Qualitatively, this spectral shape is recognisable in objects like AG Car and Romano's star. The steep $\alpha \sim 2$ slope is probably connected to the smooth shape of their flares. Yet the slope remains steep in the lowerfrequency domain, and the predicted turnover in the power spectrum has a frequency a couple of orders of magnitude higher. This may be attributed to the existing longer-time variability trends and to correlated behavior of outbursts and S Dor cycles.

Though there are indications for periodicities such as the one-year period in AG Car, a slope holding for two orders of magnitude requires some 
power-law scaling present. The duration-amplitude relation reported by van Genderen et al. (1997c) may be the key. Let us assume that the shape of a flare is determined by a single parameter $t_{f}$ (its duration), but its amplitude is $\propto t_{f}^{p}$. There are indications for $p \simeq 1$. A Poissonian series of such events will be characterised by the following power spectrum:

$$
|\tilde{F}(f)|^{2} \propto f^{-2}\left|\sum t_{f, i}^{p} \sin \left(\pi f t_{f, i}\right)\right|^{2} \simeq f^{-2(1+p)}\left|\int x^{p} \sin (\pi x) n(x / f) d x\right|^{2}
$$

Here, $n\left(t_{f}\right)=d N / d t_{f}$ is distribution of flares per unit duration time. If $p \simeq 1$, distribution of the form $n\left(t_{f}\right) \propto t_{f}^{-1}$ (uniform if one considers $\ln t_{f}$ interval) may explain the observed spectral slopes.

\section{Discussion}

\subsection{Different Kinds of Variability?}

One principal question about LBV variability is whether micro-variability (with temporal scales less than several months and amplitudes $\lesssim 0$. 2 ), S Dor cycles, slow luminosity variations and giant eruptions are produced by a single physical mechanism. As we show in this article, the basic properties of LBV variability at shorter timescales (up to $\sim 1 \mathrm{yr}$ ) may be reproduced by the toy model described in the previous section. Complex shapes of PDS at frequencies $f \sim 10^{-3} \div 10^{-2} \mathrm{~d}^{-1}$ (approximately corresponding to the duration timescale of an average flare) are possibly connected to transition from the wind-smoothed pseudo-photospheric noise at higher frequencies to a very similar Brownian noise at lower frequencies, probably connected to slow pulsations or some other internal processes.

Pulsations studied by the hydrodynamical modeling in Fadeyev (2010) are similar to the observed micro-variations in periods and amplitudes. They are also a good candidate for the process driving mass loss variations. Viewed at $\sim 1$ yr timescales, irregular pulsations are both fast and uncorrelated, that justifies the usage of white noise approximation. Longer-timescale variations may appear if some instabilities lead to secular evolution of LBV pulsations (Buchler, 1993).

Pseudo-photosphere approach is able to reproduce some of the key points of LBV variability such as the steep power spectra. Even if the higher opacity of the matter is taken into account, it is short to explain the low-frequency 
part of the PDSs. This is consistent with the conclusion of de Koter et al. (1996) who find that the observed variability of LBV stars is too strong to be explained solely by velocity and density variations in the wind.

\subsection{Variable Bolometric Luminosity}

Strongest deviations from this simple picture are the giant eruptions and "supernova impostors" (see Maund et al. (2006) for review) characterised by significant changes in bolometric luminosity (by two magnitudes and more, see for example Clark et al. (2009)). Variable bolometric luminosities are impossible to explain in the assumptions of the pseudo-photosphere approach.

Note however that variable bolometric luminosity may still arise from variations in mass loss rate if the characteristic diffusion timescale in the wind becomes large enough. Generally, the replenishment timescale (3) is much longer than the radiation diffusion timescale that may be roughly estimated as $t_{\text {diff }} \sim \tau R / c$. This makes the expanding atmosphere practically quasistationary from the point of view of energy transfer. However, if the total optical depth in the wind becomes significantly larger than the inverse wind velocity in $c$ units, $\tau>c / v \sim 10^{3}$, the energy output from the pseudophotosphere will be strongly modulated by the envelope evolution. Adiabatic losses should be taken into account as well in this case.

In these energy output modulation, there should be both increases and decreases of bolometric luminosity during the outburst. The mean level of energy release in the stellar interior is expected to be constant at much larger (thermal and nuclear) timescales of $\gtrsim 10^{4}$ yr.Bolometric luminosity should become smaller during giant eruptions because of adiabatic losses close to the photon-tiring limit (van Marle et al., 2009). During strong flares, deviations from the mean luminosity level should hold on timescales smaller than the survival time of an optically-thick outburst of the mass $M$ expelled at the maximal velocity of $v_{\text {out }}$ :

$$
t_{\text {out }} \sim \frac{1}{v_{\text {out }}} \sqrt{\frac{\varkappa_{T} M_{\text {out }}}{4 \pi}} \sim 20\left(\frac{v_{\text {out }}}{100 \mathrm{~km} \mathrm{~s}^{-1}}\right)^{-1}\left(\frac{M_{\text {out }}}{1 \mathrm{M}_{\odot}}\right)^{1 / 2} \mathrm{yr}
$$

This estimate naturally arises in a gas that remains hot. Changes in opacity and in particular gas recombination produce a variety of similar time scales proposed for the photosphere phase or plateau stage durations in supernova light curves (see for example Kasen and Woosley (2009) and references therein). 
Summarizing, we conclude that LBV eruptions should be accompanied by additional energy input inside the hydrostatic radius.

\section{Conclusions}

Variability of LBV stars is generally consistent with a single steep power law, but the processes forming its low- and high-frequency parts are probably different. The XX-th century light curve of $\mathrm{P}$ Cyg does not show any noticeable flares but is correlated at long timescales up to decades. The overall power spectrum is consistent with a $p=1.3 \div 1.4$ power law. For all the flaring sources, the slope is higher, $p \simeq 2$.

Emergence of this steep power-law spectral shape is reasonable in the pseudo-photosphere approach. Short-timescale variations in mass loss rate are effectively smeared and integrated over the line of sight that leads to a Brownian noise with a break at the "replenishment" time of about one year. Smaller amplitude variability should produce peaked noise with the maximum at several months. The resulting variability lacks two important features of the real light curves: it is not correlated at longer timescales (slow variability component) and conserves bolometric luminosity.

Longer-timescale correlations may arise partially from the higher opacity of the outflowing matter (that affects the observed luminosity changes in a complex way not implemented in our model), variable wind velocity or some internal mechanisms.

\section{Acknowledgements}

Our article makes use of the AAVSO International Database, hence we would like to thank all the AAVSO observers who made the present study possible. Author also thanks Alla Zharova for providing the V-band light curves of the two objects in M33.

\section{References}

Buchler, J.R., 1993. A dynamical systems approach to nonlinear stellar pulsations. Ap\&SS 210, 9-31.

Clark, J.S., Crowther, P.A., Larionov, V.M., Steele, I.A., Ritchie, B.W., Arkharov, A.A., 2009. Bolometric luminosity variations in the luminous blue variable AFGL2298. A\&A 507, 1555-1565. 0909.4160. 
Damineli, A., Hillier, D.J., Corcoran, M.F., Stahl, O., Levenhagen, R.S., Leister, N.V., Groh, J.H., Teodoro, M., Albacete Colombo, J.F., Gonzalez, F., Arias, J., Levato, H., Grosso, M., Morrell, N., Gamen, R., Wallerstein, G., Niemela, V., 2008. The periodicity of the $\eta$ Carinae events. MNRAS 384, 1649-1656. 0711.4250.

de Groot, M., Sterken, C., van Genderen, A.M., 2001. P Cygni: Four Centuries of Photometry, in: M. de Groot \& C. Sterken (Ed.), P Cygni 2000: 400 Years of Progress, pp. 15-+.

de Koter, A., Lamers, H.J.G.L.M., Schmutz, W., 1996. Variability of luminous blue variables. II. Parameter study of the typical LBV variations. A\&A 306, 501-+.

Fadeyev, Y.A., 2010. Instability of LBV stars against radial oscillations. Astronomy Letters 36, 362-369. 0906.0719.

Frigo, M., Johnson, S.G., 2005. The design and implementation of FFTW3. Proceedings of the IEEE 93, 216-231. Special issue on "Program Generation, Optimization, and Platform Adaptation".

Hubble, E., Sandage, A., 1953. The Brightest Variable Stars in Extragalactic Nebulae. I. M31 and M33. ApJ 118, 353-+.

Humphreys, R.M., Davidson, K., 1994. The luminous blue variables: Astrophysical geysers. PASP 106, 1025-1051.

Humphreys, R.M., Leitherer, C., Stahl, O., Wolf, B., Zickgraf, F., 1988. Variable C - an S Doradus-type variable in M 33. A\&A 203, 306-316.

Iglesias, C.A., Rogers, F.J., 1996. Updated Opal Opacities. ApJ 464, 943-+.

Kasen, D., Woosley, S.E., 2009. Type II Supernovae: Model Light Curves and Standard Candle Relationships. ApJ 703, 2205-2216. 0910.1590.

Kolka, I., 2001. Variable P Cygni: from UV to radio, in: M. de Groot \& C. Sterken (Ed.), P Cygni 2000: 400 Years of Progress, pp. 83-+.

Lamers, H.J.G.L.M., 1987. Variations in luminous blue variables, in: H. J. G. L. M. Lamers \& C. W. H. De Loore (Ed.), Instabilities in Luminous Early Type Stars, pp. 99-118. 
Markova, N., Morrison, N., Kolka, I., Markov, H., 2001. P Cygni in a short S Doradus phase. Spectroscopic and photometric evidences. A\&A 376, 898-906.

Maryeva, O., Abolmasov, P., 2010. Spectral Variability of Romano's Star. RevMexAA 46, 279-290.

Maund, J.R., Smartt, S.J., Kudritzki, R., Pastorello, A., Nelemans, G., Bresolin, F., Patat, F., Gilmore, G.F., Benn, C.R., 2006. Faint supernovae and supernova impostors: case studies of SN 2002kg/NGC 2403-V37 and SN 2003gm. MNRAS 369, 390-406. arXiv:astro-ph/0603056.

Pauldrach, A.W.A., Puls, J., 1990. Radiation-driven winds of hot stars. VIII - The bistable wind of the luminous blue variable P Cygni (B1 Ia/+/). A\&A 237, 409-424.

Polcaro, V.F., Rossi, C., Viotti, R.F., Galleti, S., Gualandi, R., Norci, L., 2011. Optical Spectrophotometric Monitoring of the Extreme Luminous Blue Variable Star GR 290 (Romano's Star) in M 33. AJ 141, 18-+.

Press, W.H., Rybicki, G.B., 1989. Fast algorithm for spectral analysis of unevenly sampled data. ApJ 338, 277-280.

Sholukhova, O., Zharova, A., Fabrika, S., Malinovskii, D., 2002. Complex Pulsation-Like Variability of the LBV Star v 532 in M 33, in: C. Aerts, T. R. Bedding, \& J. Christensen-Dalsgaard (Ed.), IAU Colloq. 185: Radial and Nonradial Pulsationsn as Probes of Stellar Physics, pp. 522-+.

Terebizh, V.Y., Terebizh, A.V., Biryukov, V.V., 1989. Flicker-noise model for optical fluctuations of NGC 4151. Astrophysics 31, 460-467.

van Genderen, A.M., 1985. An investigation of the micro-variations of highly luminous OBA type stars. II. A\&A 151, 349-360.

van Genderen, A.M., de Groot, M., Sterken, C., 1997a. New perceptions on the $S$ Doradus phenomenon and the micro variations of five Luminous Blue Variables (LBVs). A\&ASS 124, 517-531.

van Genderen, A.M., Sterken, C., de Groot, M., 1997b. New discoveries on the $\mathrm{S}$ DOR phenomenon based on an investigation of the photometric history of the variables AG Car, S DOR and Eta Car. A\&A 318, 81-98. 
van Genderen, A.M., Sterken, C., de Groot, M., 1997c. New discoveries on the $\mathrm{S}$ DOR phenomenon based on an investigation of the photometric history of the variables AG Car, S DOR and Eta Car. A\&A 318, 81-98.

van Marle, A.J., Owocki, S.P., Shaviv, N.J., 2009. On the behaviour of stellar winds that exceed the photon-tiring limit. MNRAS 394, 595-604. 0812.0242 . 